\title{
Constrained Optimization of Resource Allocation in Colleges
}

\author{
Mengdie Ye \\ School of North China Electric Power University (Baoding), Baoding 071000, China \\ yemengdie727@163.com
}

Keywords: Constrained Optimization, Resource Allocation, Genetic Algorithms.

\begin{abstract}
In this thesis, the resource allocation model based on Immune Clone Algorithms, highly conforming to reality, brings various complicated factors in actual course arrangement into model computing. The solution shows that the model can effectively arrange courses in colleges and universities, which deserves popularization and application.
\end{abstract}

\section{Introduction}

Course arrangement is a constrained and multi-objective combinational optimization problem. It's impossible to find adequate constraint conditions due to a great deal of soft constraints of the course arrangement. For the new multi-objective optimization problem, solutions will be a set called feasible solution set. In this model, besides meeting all the hard and newly-added constraint conditions, it just needs course arrangement to be rational and practical, irrespective of soft constraint conditions.

\section{Model}

\subsection{Summary.}

According to the course timetable target and its mathematical model, this thesis also divides the course arrangement into two steps:

a. Random course arranging operation according to the teaching assignment book by using initial population to generate the algorithms;

b. Using immune algorithm to optimize the generated random feasible course arrangement scheme.

\subsection{Coding scheme.}

Since the set of courses, classes and teachers are known, so we can think it as a tuple called class tuple. In 3-dimensional coding, $X$ axis represents time and each time coordinate corresponds to a time quantum. $\mathrm{Y}$ axis represents a classroom and $\mathrm{Z}$ axis represents a class tuple. Hence, one 3-dimensional coordinate reflects the arrangement of one course of one class.

To ensure feasible solution for one problem, if $\mathrm{X}$ axis and $\mathrm{Y}$ axis are certain, there are 5 time quanta and 5 school days in total. Among them, compulsory courses can be arranged within time quanta 1, 2 and 3, but in time quanta 4 and 5 no courses can be arranged. Hence, there exists:

$$
\left\{\begin{array}{l}
\sum_{i=1}^{n} x y z_{i} \leq 1(n=15) \\
\sum_{i=16}^{n} x y z_{i}=1(n=25)
\end{array}\right.
$$

For the solution of models, only one constraint condition is added to the basic model. So, except this constraint condition, other model computations are alike to the basic model.

(1)Generating scheme of initial antibody population

Also supposing that $\mathrm{i}, \mathrm{j}, \mathrm{k}$ are teacher, time and classroom respectively. Dealing according to the newly-added constraint conditions and converting to non-constraint problem. In addition, in the actual course arrangement, we can extract immune vaccine. Through the vaccine, we can utilize prior information effectively, overcome the blindness of algorithms and speed up searching.

(2)Immune selection based on the vaccine receiver concentration

To avoid premature convergence of immune algorithms and for the purpose of occupying least classrooms, we can set a constraint condition that all the classroom have their highest usage rate. The higher the ratio between the student number having class in one classroom and the capacity number 
of that classroom is, the higher the rate of resource utilization is. The maximum ratio is 1 , meaning the numbers just match with each other. Optimal object:

$\operatorname{Max}(f)=\Sigma$

(3)Immune self-adaptive mutation

The selection of mutation probability largely affect the convergence rate of the algorithms and the solution's quality. This thesis adopts the strategy of self-adaptive mutation.

(4)Steps for Immune Clone Algorithms

Basic steps as follows:

a. Initializing the population randomly, recording the scale as setting memory population, with a blank beginning. Each antibody is a course timetable and a course arrangement scheme. At the same time initializing the population,

b. Evaluating the affinity function. Storing the antibodies with higher affinity into the memory unit.

c. Judging the terminal conditions; terminating the algorithms and outputting the results if reaching the biggest iteration $\mathrm{N}$; if not, turn to step (4).

d. Clone mutation; Adopting clone mutation toward antibodies with high affinity. Clone here requires equal proportion. The mutation strategy is keeping more clones of antibodies with low concentration and high affinity in order to keep the diversity of the antibodies and avoid premature convergence.

e. Clone selection. The selection adopts the selecting probability computing method of similarity vector distance, and turn to step (2).

\subsection{Simulation experiment and analyses.}

a. Experiment data and parameter setting

The experiment data is from the listed curriculum information in the attachment. In the immune algorithms of this thesis, we set the population scale as 100 , memory unit scale as $0.4 \mathrm{x}$, control parameter of clone scale $\mathrm{n}$ as 20, the biggest iteration $\mathrm{N}$ as 1000 and no setting to mutation probability with self-adaption. Under the circumstances of Window, the algorithms work through Matlab programming.

\section{b. Experiment results}

The experiment adopts fitness function, average time cost in evolution and the effect of course arrangement as measurement indexes. To avoid randomness of one experiment result, the experiment have been conducted for 10 times and takes the average optimal fitness value as the experiment result. Recording one optimal fitness value (the optimal solution) every 100 iterations, as shown in Figure 1. Recording a time point every 100 iterations and taking the average of the 10 times' records as the experiment result of the time.

\subsection{Solving of the course arrangement after constraining actual needs.}

Course arrangement is a constrained and multi-objective combinational optimization problem. It's impossible to find adequate constraint conditions due to a great deal of soft constraints of the course arrangement. By using the data from attachment 1 and 2, new constraint conditions will be added to this model.

(1)Arranging as fewer compulsory courses as possible in time quanta 4 and 5;

(2)Arranging as fewer courses with as possible in time quanta 1 and 5;

(3)Arranging as fewer courses as possible in time quanta 4 and 5 on every Friday;

For the new multi-objective optimization problem, solutions will be a set called feasible solution set. In this model, besides meeting all the hard and newly-added constraint conditions, it just needs course arrangement to be rational and practical, irrespective of soft constraint conditions.

\subsection{Coding scheme.}

To ensure feasible solution for one problem, if $\mathrm{X}$ axis and $\mathrm{Y}$ axis are certain, there are 5 time quanta and 5 school days in total, there exists: 


$$
\left\{\begin{array}{c}
\sum_{i=1}^{n} x y z_{i} \leq 1(n=15) \\
\sum_{i=16}^{n} x y z_{i}=0(n=25) \\
\sum_{i=1}^{23} x y z \leq 1 \\
\sum_{i=24}^{25} x y z_{i}=0
\end{array}\right.
$$

For the solution of models, only one constraint condition is added to the basic model. So, except this constraint condition, other model computations are alike to the basic model.

\section{Conclusion}

The experiment adopts fitness function, average time cost in evolution and the effect of course arrangement as measurement indexes. To avoid randomness of one experiment result, the experiment have been conducted for 10 times and takes the average optimal fitness value as the experiment result. Recording one optimal fitness value (the optimal solution) every 100 iterations, as shown in Figure 1. Recording a time point every 100 iterations and taking the average of the 10 times' records as the experiment result of the time.

\section{References}

[1]. Pillay N, Banzhaf W. An informed genetic algorithm for the examination time tabling problem[J]. Applied Soft Computing 2010, 10(2): 457-467.

[2]. Li Wang, Research and design of course scheduling system based on Immune Clonal Algorithm [D]. Fudan University, 2011.

[3]. Jiandong Zhu, Weiju Jiang. The Immune Clone Algorithm scheme based on schedule arrangement [J]. Computer Project, 2011, (22). 